\title{
RIFAMPICIN LOADED SPRAY-DRIED OLIBANUM GUM RESIN PULMOSPHERES FOR LUNG DELIVERY
}

\author{
Patil J.S. ${ }^{1}$, Jagadhane V. B. ${ }^{1}$, Jamagondi L.N ${ }^{2}$, Gurav P.B. ${ }^{1}$, \\ ${ }^{1}$ Dept. of Pharmaceutics, SVERI's College of Pharmacy, Gopalpur-Ranjani Road, Gopalpur, Pandharpur-413 304, \\ Maharashtra, India. \\ ${ }^{2}$ Dept. of Pharmaceutics, DSTSM's College of Pharmacy, Jule Solapur, Vijapur Road, Solapur-413 004, Maharashtra, \\ India. \\ *Corresponding author's email: pharmajspatil@gmail.com
}

\begin{abstract}
Pulmospheres of Rifampicin were prepared successfully with cyclodextrin using olibanum gum resin as a rate controlling biodegradable natural polymer by novel spray drying technique. Hydroxypropyl- $\beta$-cyclodextrin was used as solubilising agent to enhance the solubility and permeation of poorly soluble rifampicin. The physcochemical characteristics of rifampicin loaded pulmospheres were evaluated for mean particle size, thermal behaviour and surface morphology. The mean particle size for optimized formulation F1 was found to be $3.5 \mu \mathrm{m}$ and spray-dried pure rifampicin also showed same value. Scanning Electron Microphotographs of pure drug has shown a typical and regular size indicated crystalline nature, whereas, spray dried pure drug and formulation F1 have shown spherical particles with porous surface indicated the conversion of crystalline drug into amorphous form. Rifampicin loaded spray-dried pulmospheres using olibanum gum resin showed drug release up to 72 hours. The release of rifampicin was greater in simulated physiological fluid of $\mathrm{pH} 7.4$ which was $94 \%$ as compared to simulated lung fluid of $\mathrm{pH} 5.2$ at the end of 72 hours. Rifampicin loaded spray-dried microspheres were proposed for pulmonary drug delivery.
\end{abstract}

Keywords: Rifampicin, Lung targeting, Olibanum gum resin, Spray-drying, Pulmospheres

\section{INTRODUCTION}

Tuberculosis (TB) is emerging as a major health concern day by day. TB is a communicable chronic bacterial infection and still a leading human killer worldwide ${ }^{1}$. Each year, approximately 2 million persons worldwide die and 9 million become infected ${ }^{1}$. TB has returned with a new face and the global scourge multidrug resistant TB (MDR-TB) is reaching epidemic proportions. ${ }^{1}$ The prevalence of $\mathrm{TB}$ is continuing to increase because of the increased number of patients infected with human immunodeficiency virus, bacterial resistance to medications, increased international travels and immigration from countries with high prevalence, and the growing numbers of homeless and drug abusers. ${ }^{2}$ Mycobacteria grow 20-100 times slower than other bacteria and it take 4-6 weeks to obtain a colony for drug sensitivity studies. TB is treated with a multidrug regimen, and is thus exceptionally vulnerable to incidences of side effects, unsatisfactory patient compliances and slow improvement of patients. ${ }^{3}$ TB germs usually spread through air. When a patient with pulmonary TB coughs or sneezes, germs are spread in the air in the form of tiny droplets ${ }^{4}$ and such droplets when inhaled by a healthy person are then taken up by alveolar macrophages, initiating a cascade of events that result in either successful containment of the infection or progression to active disease (primary progressive TB). ${ }^{5}$ The current treatments of tuberculosis are limited by their methods of delivery. Oral bioavailability of rifampicin (RIF) is $90-95 \%{ }^{6}$ and the doses above those currently administered would present the risk of toxic side effects. Targeting anti-tubercular drugs such as RIF to the lung may increase the local therapeutic effect and reduce systemic exposure ${ }^{7}$. Pulmonary drug delivery system is gaining popularity as it is possible to achieve local and systemic delivery of drugs directly to lungs for the treatment of chronic obstructive pulmonary diseases and other respiratory diseases. Since last decade sustained release microspheres have been proposed for pulmonary delivery with major advantage of avoiding systemic toxicity by local delivery of the drug. ${ }^{8-9}$ Due to chronic oral systemic usage of presently available anti-tubercular agents produces patient noncompliance and systemic side effects. ${ }^{10}$ The problems and issues surrounding the treatment of TB infections and disease are more complex because of body defense appears to have little effect on the bacteria and poor lung permeation of available drugs. ${ }^{11}$ Pulmonary delivery of drug has become an attractive target and of tremendous scientific and biomedical interest in the health care research area as the lung is capable of absorbing pharmaceuticals either for local deposition or for systemic delivery. ${ }^{12}$ It is one of the most non-invasive approach of drug delivery to the lung by which unwanted systemic absorption of 
drugs delivered for the local treatment of respiratory disease can be reduced. ${ }^{13-14}$ The high frequency of pulmonary tuberculosis demands the development of novel drug delivery approaches that enhances the bioavailability of the drug at the level of lungs. ${ }^{15}$

Enhancement in solubility and permeation of poorly soluble drugs in cyclodextrin inclusion complex form has been reported. Cyclodextrins act as permeation enhancers by carrying the drug through the aqueous barriers which exists before the lipophilic surface of biological membranes. ${ }^{16}$ Sugars and cyclodextrins though compatible with lung can cause bronchoconstriction in many of the hypersensitive individuals ${ }^{12}$. Olibanum resin forms an excellent coat material in the resin coated microcapsules and exhibited good controlled release characteristics. Hence, the resin can be utilized as a matrix forming substance in the formulation of matrix tablets and film forming coating polymer for designing the microcapsules which can facilitate to attain once a day oral controlled release products. ${ }^{17}$ The Hydroxypropyl- $\beta$-cyclodextrin (HP $\beta C D)$ with Olibanum gum resin containing RIF may be delivered to lungs by pulmonary route to improve the solubility and permeation of drug to achieve better treatment of TB. The lung comtatibilty and safty aspects of olibanub gum resin a natural polymer is yet to established. Hence present study was aimed to design microspheres of RIF with HP $\beta C D$ by using extracted olibanum gum resin by a novel spray-drying technique for improved therapeutic effect of poorly soluble RIF for lung delivery.

\section{MATERIALS AND METHODS}

RIF was obtained as generous gift sample from Lupin
Pharmaceuticals, Mumbai, India. Olibanum Gum was purchased from Girijan Co-operative Ltd., Vishakhapattanum, Andhra Pradesh. HPßCD was obtained as gift sample from Gangwal chemicals, Mumbai, India. All other chemicals used were of analytical grade and were used without further purification.

\section{Extraction of resin fraction from Olibanum gum}

The resin present in powdered Olibanum gum (10 gm) was extracted repeatedly with $4 \times 50 \mathrm{ml}$ quantities of solvent ether. The ether extracts were collected in a porcelain dish and concentrated to dryness at $40^{\circ}$ to obtain the resin fraction. The dry mass was powdered and the size was reduced to 200 mesh, packed in aluminium foil and stored in a deccicator till further use. $^{17}$

\section{Preparation of spray-dried microspheres ${ }^{18}$}

Olibanum gum resin and RIF were dissolved one after another in $475 \mathrm{ml}$ of Isopropyl alcohol with continuous stirring to get a clear homogeneous solution. HP $\beta C D$ was dissolved in another beaker containing $25 \mathrm{ml}$ distilled water to get a clear homogeneous solution. Solution of HP $\beta C D$ was added slowly to resin and RIF solution and mixed together on a magnetic stirrer until a homogeneous solution was formed. Final solution thus formed was spray-dried using a mini spray-dryer (LabUltima-222, Mumbai) at the following predetermined conditions; nozzle diameter- $0.7 \mathrm{~mm}$, atomization pressure- $1.5 \mathrm{~kg} / \mathrm{cm}^{2}$, feed rate $5 \mathrm{ml} / \mathrm{min}$, aspirator- $45 \%$, inlet temperature- $70^{\circ}$ and outlet temperature $40^{\circ}$. The details of various formulation compositions are presented in Table 1.

Table 1: Formulation details of RIF loaded pulmospheres

\begin{tabular}{ccccc}
\hline Batch code & RIF & Olibanum Resin & HPßCD & Isopropyl Alcohol \\
\hline F1 & $5 \mathrm{gm}$ & $400 \mathrm{mg}$ & $5 \mathrm{gm}$ & $500 \mathrm{ml}$ \\
F2 & $5 \mathrm{gm}$ & $800 \mathrm{mg}$ & $2.5 \mathrm{gm}$ & $500 \mathrm{ml}$ \\
F3 & $5 \mathrm{gm}$ & $1200 \mathrm{mg}$ & $1.25 \mathrm{gm}$ & $500 \mathrm{ml}$ \\
F4 & $5 \mathrm{gm}$ & $800 \mathrm{mg}$ & - & $500 \mathrm{ml}$ \\
F5 & - & $800 \mathrm{mg}$ & $2.5 \mathrm{gm}$ & $500 \mathrm{ml}$ \\
\hline
\end{tabular}

\section{Evaluation and Characterization of Spray dried microspheres}

\section{Drug content ${ }^{19}$}

Known amount of crushed pulmospheres equivalent to $50 \mathrm{mg}$ of RIF were added to $10 \mathrm{ml}$ of methanol in a centrifuge tube, kept overnight at $37^{\circ}$ for complete swelling and extraction of drug. The content was centrifuged to remove polymeric debris. The clear supernatant solution was diluted suitably with phosphate buffer of pH 5.2 and $\mathrm{pH} 7.4$ separately and analysed for drug content using UV-Visible spectrophotometer (Lab India 3200) at $475 \mathrm{~nm}$.

\section{Particle Size analysis}

Particle Size Analysis was performed using Beckman Coulter, Delsa Nano (common) India. The particles of optimized formulation F1 and spray-dried pure RIF were dispersed separately in water and the mean particle size was determined.

\section{In- vitro dissolution studies ${ }^{20-21}$}

Spray-dried pulmospheres equivalent to $50 \mathrm{mg}$ of drug were dispersed in $50 \mathrm{ml}$ of dissolution media (7.4 physiologic $\mathrm{pH}$ and 5.2 endosomal of alveolar macrophages $\mathrm{pH}$ ) separately. The dissolution study was performed both in simulated normal physiological medium and simulated lung fluid to compare the drug release pattern in both the media. The test tubes were maintained at $37^{\circ}$ in a horizontal-shaking bath and shaken at a rate of 90 cycles/min. At predetermined intervals, $1 \mathrm{ml}$ of the sample was withdrawn and filtered. The medium was replaced with fresh buffer solutions to maintain the total volume throughout the study. 200 $\mu \mathrm{g} / \mathrm{ml}$ ascorbic acid was added as an antioxidant to prevent oxidative degradation. The concentration of 
rifampicin was determined by UV spectrophotometer at $475 \mathrm{~nm}$.

\section{Differential Scanning Calorimetry (DSC)}

DSC was performed on DSC D60 Shimadzu, Japan. About $5 \mathrm{mg}$ of samples was sealed in aluminium pans and heated at the rate of $10 \% \mathrm{~min}$. Covering temperature range was between $40-300^{\circ}$ under nitrogen atmosphere with a flow rate of $100 \mathrm{ml} / \mathrm{min}$.

\section{Scanning Electron Microscopy (SEM) ${ }^{22}$}

The surface morphology of the samples was investigated using scanning electron microscope (Jeol, JSM-6360, Germany). SEM studies help to observe changes that occur in crystal structure during or after preparation. The samples were mounted onto stubs with double sided adhesive tape and sputter coated with coated with gold using a gold coater the instrument. The coated sample was observed under SEM instrument at the required magnification at room temperature.

\section{RESULTS AND DISCUSSION}

\section{Percentage yield, Drug content and Particle size}

The results of percentage yield, drug content and mean particle size of spray-dried pulmospheres are mentioned in table 2. Percentage yield of all the formulations was found to be in the range of 48-50. No significant difference in the percentage yield of all the formulations was found. The drug content of all spray dried pulmospheres in both $\mathrm{pH} 5.2$ and in $\mathrm{pH} 7.4$ was found to be in the range of $98 \%-100 \%$. The drug content was found to $100 \%$ in case of SDPD whereas it was found to be $99 \%$ for F1 and F2. There was no significant difference in drug content was found between all the formulations. Mean particle size of spray-dried pure drug (SDPD) and Formulation F1 was found to be $3.5 \mu \mathrm{m}$ which was similar in both the cases and spray-dried particles were in the respirable size range suitable to administer through pulmonary route. The intensity distribution curves are shown in Figure 6 and 7.

Table 2: Preliminary evaluation data of RIF loaded pulmospheres

\begin{tabular}{|c|c|c|c|c|}
\hline \multirow{2}{*}{ Batch Code } & Percentage Yield & \multicolumn{2}{|c|}{ Drug content } & Mean Particle Size( $\boldsymbol{\mu m})$ \\
\cline { 3 - 5 } & & In pH 5.2 & In pH 7.4 & \\
\hline SDPD & $50 \%$ & $100 \% \pm 0.60$ & $100 \% \pm 0.40$ & 3.5 \\
\hline F1 & $50 \%$ & $99 \% \pm 0.20$ & $99 \% \pm 0.90$ & 3.5 \\
\hline F2 & $48 \%$ & $99 \% \pm 0.90$ & $99 \% \pm 0.50$ & --- \\
\hline F3 & $49 \%$ & $98 \% \pm 0.40$ & $98 \% \pm 0.60$ & --- \\
\hline F4 & $48 \%$ & $98 \% \pm 0.70$ & $99 \% \pm 0.20$ & --- \\
\hline
\end{tabular}

\section{In Vitro drug release}

In-vitro dissolution study was carried by modified dissolution apparatus (rotator shaker method). The release profiles are shown in Figure 1 and 2. The drug release study was carried in both $\mathrm{pH} 5.2$ and in $\mathrm{pH} 7.4$ for up to $72 \mathrm{~h}$. The drug release from formulation F1 was highest in both the $\mathrm{pH}$ due to the presence of HP $\beta C D$. As the amount of HP $\beta C D$ increased, solubility of RIF was also increased proportionally. Hence, the drug release was also found to be high. The cumulative release of RIF significantly decreased with increasing polymer concentration. The dissolution profile of drug from formulation containing olibanum resin in presence of $\mathrm{HP} \beta \mathrm{CD}$ was improved as compared to pure drug. However, dissolution was greater in physiological media as compared to lung media in all the cases. The drug release from the formulation containing resin as a rate retarding material in presence of $\mathrm{HP} \beta C D$ has sown enormous improvement when compared to pure drug alone which has shown only $28 \%$ release. There was 3 fold increases in dissolution rate found in $\mathrm{pH} 5.2$ media. The drug release from SDPD was more as compared to pure drug in both dissolution media.

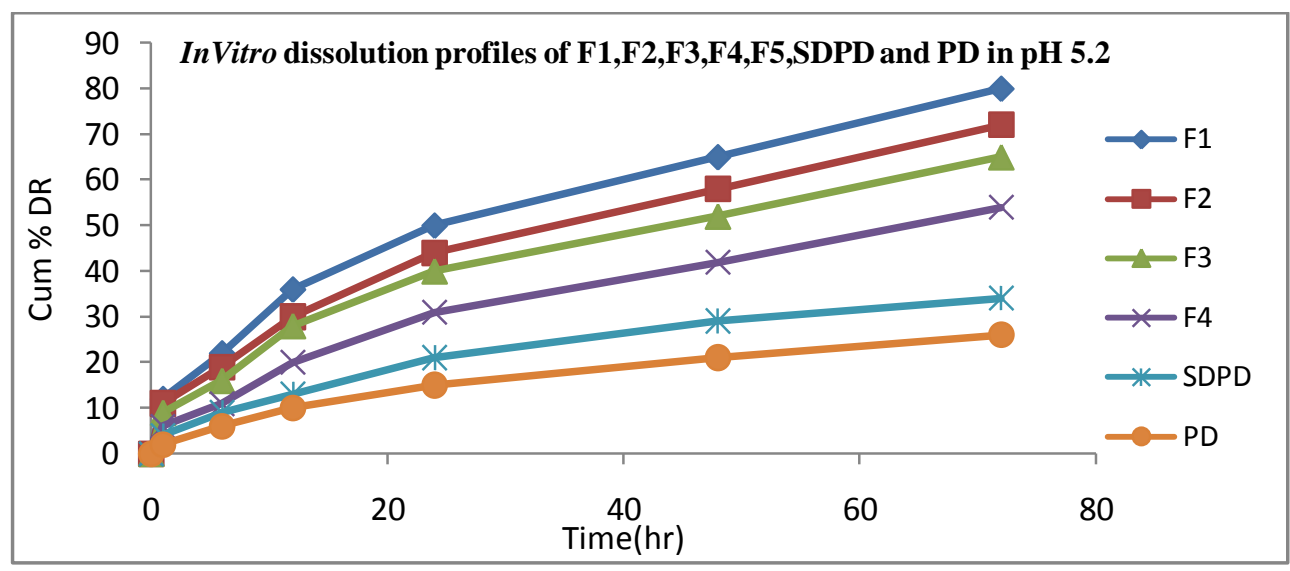

Figure 1: In Vitro dissolution profile of F1, F2, F3, F4, SDPD and PD in pH 5.2 


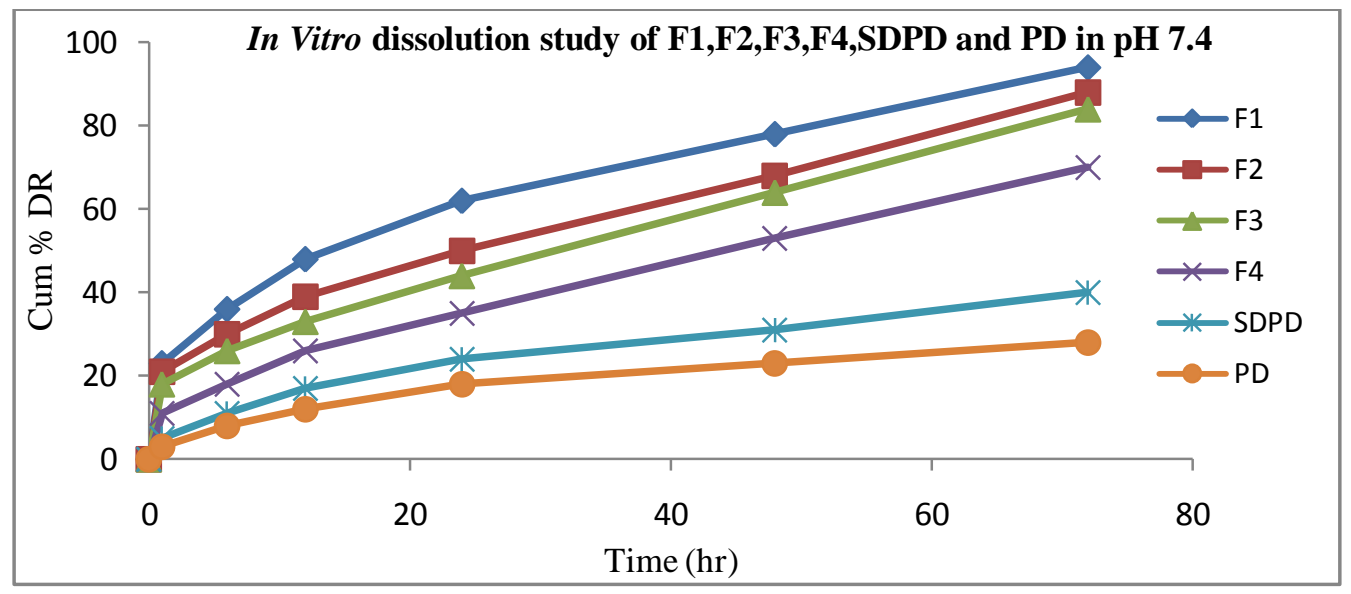

Figure 2: In Vitro dissolution profile of F1, F2, F3, F4, SDPD and PD in pH 7.4

Characterisation of spray dried microspheres

\section{Differential Scanning Calorimetry (DSC)}

DSC thermograms of pure RIF and optimized formulation $\mathrm{F} 1$ are shown in figures 3 and 4 respectively. The DSC thermogram of pure rifampicin has shown endothermic peak at $198^{\circ}$ which is due to melting of drug. The thermogram of F1 has shown broad endothermic peak near the melting point of drug that indictes the complete encapsulation of drug into the polymer matrix without degradation.

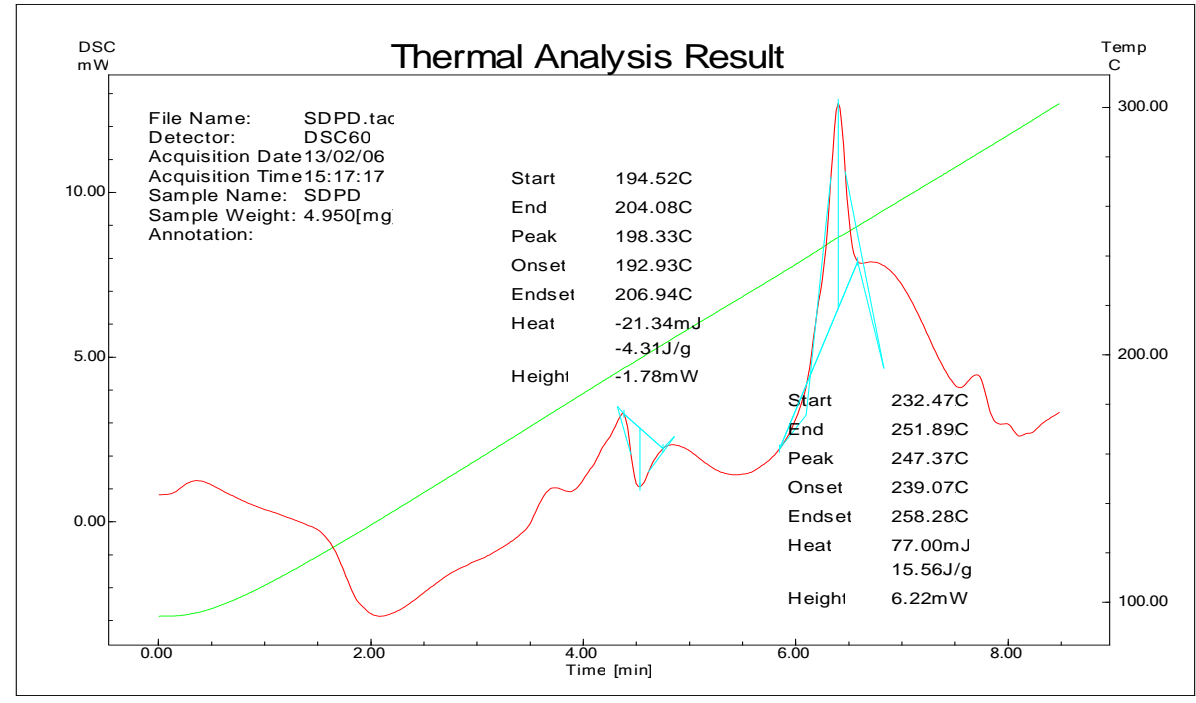

Figure 3: DSC Thermograms of pure Rifampicin

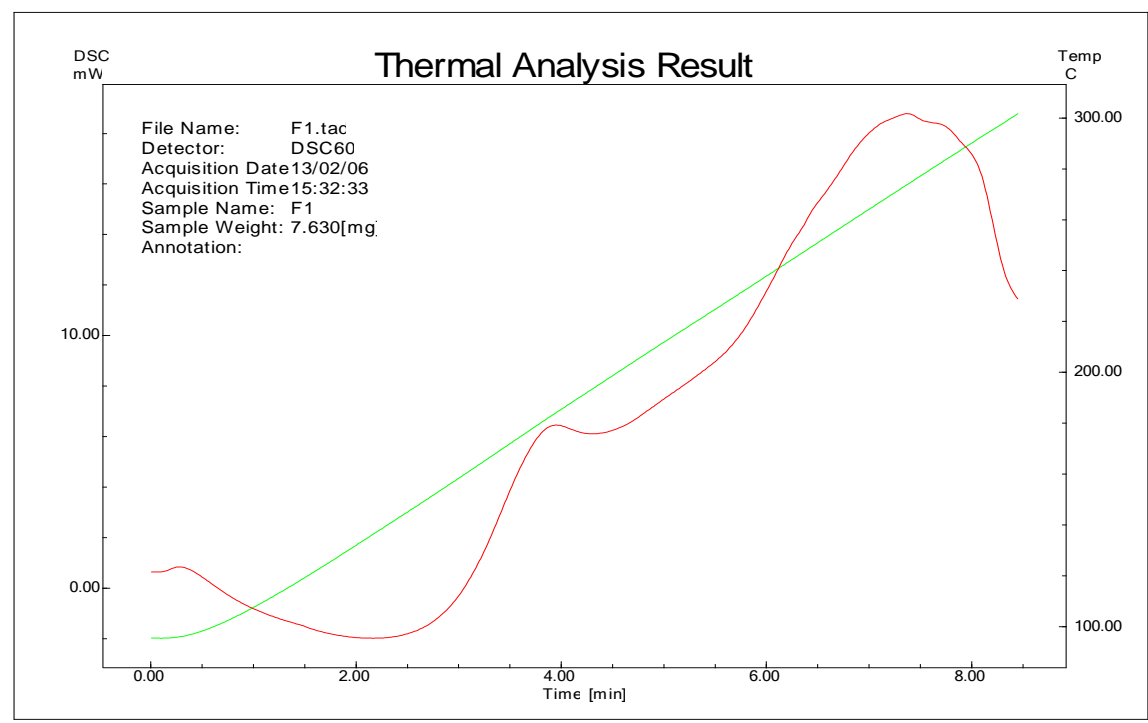

Figure 4: DSC Thermogram of formulation F1 


\section{Scanning Electron Microscopy (SEM)}

The SEM of pure RIF, Spray-dried pure RIF, optimized formulation $\mathrm{F} 1$ and $\mathrm{HP} \beta \mathrm{CD}$ were shown in figure 5 . The SEM study revealed that, pure drug has shown more crystalline nature of its typical and regular size, whereas,
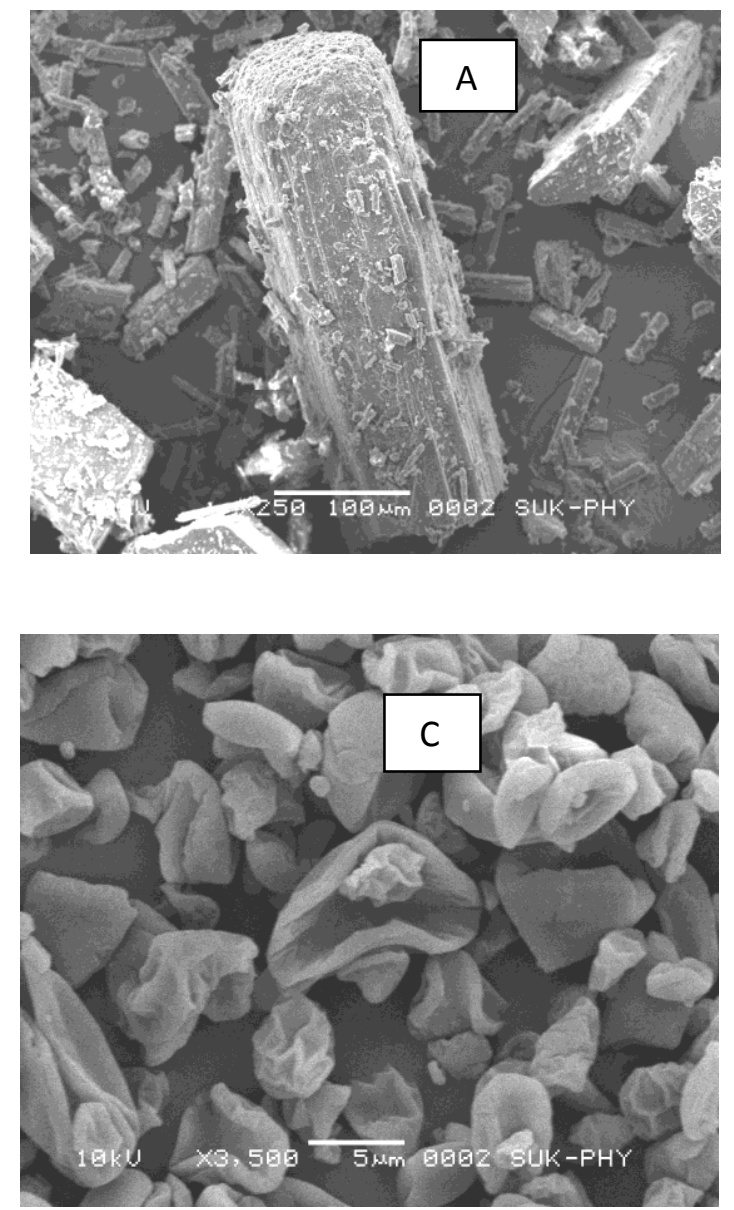

spray dried pure drug has shown lesser crystals and there was a formation of spherical drug particles having porous morphology. The formulation F1 shows smooth surface having folding and curves over it indicating the formation of perfect inclusion complex with HPßCD and complete encapsulation of drug in polymers matrix.
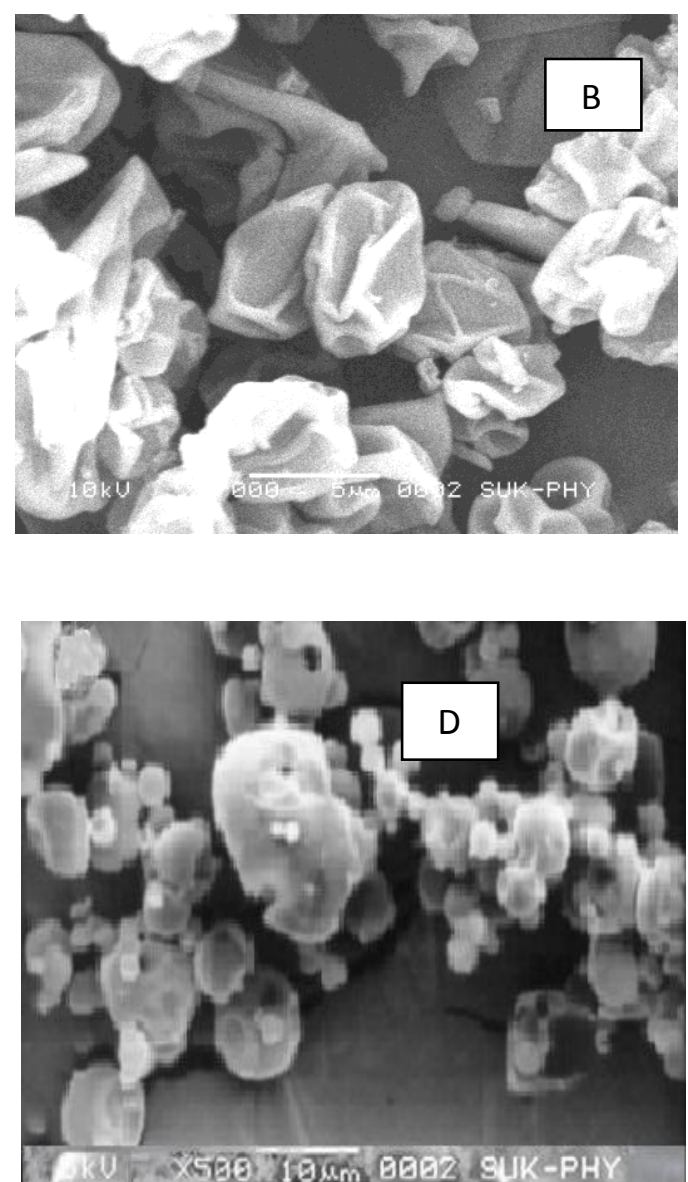

Figure 5: SEM microphotographs of pure Rifampicin (A), Spray dried pure Rifampicin (B), Formulation F1(C) and HPßCD (D)

\section{CONCLUSION}

This study reports for the first time the effect of cyclodextrin as a solubility enhancer in the sustained release rifampicin loaded pulmosphere formulation designed using naturally available, biocompatible olibanaum gum resin pulmosphere as a tare retarding polymer in order to improve its bioavailability for

\section{REFERENCES}

1. Lisa CT, Viness P, Michael PD. Tuberculosis chemotherapy: current drug delivery approaches, Resp Res, 2006, 7, 118

2. Goldrick BA. Once dismissed, still rampant: tuberculosis, the second deadliest infectious disease worldwide, Am J Nurs, 2004, 104(90), 68-70.

3. Prabakaran D, Singh P, Jaganathan KS, Vyas SP. Osmotically regulated asymmetric capsular systems for simultaneous sustained delivery of anti-tubercular drugs, J Control Rel, 2004, 95, 239-48.

4. Koul A, Arnoult E, Lounis N, Guillemont J, Andries K. The challenge of new drug discovery for tuberculosis Nature, 2011, 469-83.

5. http://respiratory-research.com/content/7/1/118.

6. Kenny MT, Srates B. Metabolism and pharmacokinetics of the antibiotic rifampicin, Drug Metab, Rev, 1981, 12, 159218. effective tuberculosis control. The developed pulmospheres demonstrated approximately 3 fold increased drug release compared with pure drug alone. The presence of cyclodextrin helped to achieve higher solubility and dissolution of rifampicin and olibanum gum resin was found to be a suitable rate retarding polymer to achieve desired sustained release of drug. 
11. David S. Tuberculosis and non- tuberculosis mico-bacterial infections. 4th ed. Philadelphia; W. B. Saunders's company. 1971. 143.

12. Patil JS, Sarasija S. Pulmonary Drug Delivery Strategies: A concise, systematic review, Lung India, 2012, 29, 44-9.

13. Zahoor A, Sharma S, Khullar GK, Inhalabale alginate nanoparticles as anti-tubercular drug carriers against experimental tuberculosis, Int J Antimicrob Agent, 2005, 26, 298-303.

14. Gonda I, Systemic delivery of drugs to human via inhalation, J Aerosol Med, 2006, 19, 47-53.

15. Patil JS, Sarasija S, Physicochemical Characterization, invitro release and permeation studies of respirable rifampicimcychlodextrin Inclusion Complexes, Indian J Pharm Sci, 2009, 71(6), 638-43.

16. Prakashrao B, Sarasija S, Narendra C, Recent advances in cyclodextrin complexes: Mechanisticanalysis as permeation enhancer, Indian J Pharm Edu Res, 2007, 41, 102-13.

17. Patil JS, Kadam DV, Marapur SC, Kamalapur MV, Pharmaceutical and medicinal applications of olibanum gum and its constituents: A review, J Pharm Res 2010, 3, 587-89.
18. Patil JS, Kadam DV, Ssiralshetti SS, Marapur SC, Kamalapur MV, Utilization of natural olibanum gun resin as a rate controlling polymer in design and evaluation of microspheres of an antiretroviral drug by using a unique spray drying technique, Indian J Pharm Edu Res, 2012, 46(2), 155-60.

19. Smrdel P, Bogataj M, Mrhar A, The influence of selected parameters on the size and shape of Alginate beads prepared by ionotropic gelation, Sci Pharm, 2008, 76, 77-89.

20. Hara PO, Hickey AJ, Respirable PLGA microspheres containing Rifampicin for the treatment of tuberculosis: Manufacture and characterisation, Pharm Res, 2000, 17, 95561.

21. Davis NM, Feddah MR, Anovel method for assessing dissolution of aerosol inhaler products, Int J Pharm, 2003, 255, 175-87.

22. Jain NK. Progress in controlled and novel drug delivery systems: CBS Publications; 2004. 391. 